\title{
Alterations in Fetal Leydig Cell Gene Expression during Fetal and Adult Development
}

\author{
Kanako Miyabayashi ${ }^{a}$ Yuichi Shima ${ }^{a, b, f}$ Miki Inoue $^{b}$ \\ Tetsuya Sato ${ }^{b, c, e}$ Takashi Babaa, ${ }^{a}$ Yasuyuki Ohkawa ${ }^{b, d, e}$ \\ Mikita Suyamab, c, e Ken-ichirou Morohashia, b \\ a Department of Molecular Biology, Graduate School of Medical Sciences, ${ }^{b}$ Department of Systems Life Sciences, \\ Graduate School of Systems Life Sciences, and ${ }^{\mathrm{C}}$ Division of Bioinformatics and ${ }^{\mathrm{d}}$ Research Center for Transomics \\ Medicine, Medical Institute of Bioregulation, Kyushu University, and ${ }^{\mathrm{e}}$ AMED-CREST, Japan Agency for Medical \\ Research and Development, Fukuoka, and ${ }^{\mathrm{f}}$ Department of Anatomy, Kawasaki Medical School, Okayama, Japan
}

\section{Key Words}

Gene expression · Leydig cells · Metabolism .

Steroidogenesis

\begin{abstract}
Fetal Leydig cells (FLCs) and adult Leydig cells (ALCs) develop in the mammalian prenatal and postnatal testes, respectively. In mice, FLCs emerge in the interstitial space of the testis as early as embryonic day 12.5 and thereafter increase in number during the fetal stage. We previously established a transgenic mouse line in which FLCs are labeled with EGFP and demonstrated that the EGFP-labeled FLCs were present even in adult testes. However, the characteristics of FLCs during postnatal stages remained unclear. In the present study, a comparison of the transcriptomes of FLCs from prenatal and postnatal testes and of ALCs from adult testes revealed that FLCs gradually alter their characteristics across developmental stages and come to roughly resemble ALCs. Many cholesterogenic genes simultaneously expressed a
\end{abstract}

\section{KARGER}

(C) 2016 S. Karger AG, Basel

E-Mail karger@karger.com

www.karger.com/sxd unique alternation pattern, while many oxidative phosphorylation and $\beta$-oxidation (both mitochondrial functions) genes showed a different unique pattern. These metabolic gene expression alterations might be triggered by milieu changes, such as nutrient and oxygen supply, from the prenatal to the postnatal period.

(c) 2016 S. Karger AG, Basel

After Sertoli cell differentiation is induced by the transient expression of the male-determining SRY gene, the testes are structurally divided into 2 compartments, the testicular cords and the interstitial space. While Sertoli and germ cells are localized within the testicular cords, several cell types develop in the interstitial space [Brennan and Capel, 2004; Svingen and Koopman, 2013]. Among these, Leydig cells have been well characterized in terms of their ability to produce androgen, which is essential for masculinization and maintenance of malespecific characteristics. 
Two types of Leydig cells, fetal Leydig cells (FLCs) and adult Leydig cells (ALCs), develop in mammalian prenatal and postnatal testes, respectively [Griswold and Behringer, 2009; Teerds and Huhtaniemi, 2015]. The mechanisms underlying the differentiation of FLCs have been studied extensively, and the involvement of transcription factors [Meeks et al., 2003; Cui et al., 2004; Buaas et al., 2012; van den Driesche et al., 2012; Miyabayashi et al., 2013] and growth factors [Yao et al., 2002; Brennan et al., 2003; Tang et al., 2008] has been determined. Both Leydig cell types display morphological and functional differences in addition to developmental timing; interestingly, this includes the capability for steroid production. While ALCs synthesize testosterone from cholesterol, FLCs cannot synthesize testosterone due to the absence of $17 \beta$-hydroxysteroid dehydrogenase type 3 (HSD17B3), which mediates the reaction from androstenedione to testosterone. HSD17B3 is expressed in Sertoli cells in fetal testes; hence, testosterone is produced by the cooperative action of FLCs and Sertoli cells during the fetal period [O'Shaughnessy et al., 2000; Shima et al., 2013].

While many studies have described the disappearance of FLCs soon after birth, a few studies have reported the persistent presence of FLCs in postnatal testes [Kerr and Knell, 1988; Kuopio et al., 1989]. However, this observation seems to have been overlooked or underestimated, probably because these studies were dependent on morphological evidence. Recently, the persistent presence of FLCs in postnatal testes was directly demonstrated by the lineage tracing analyses with the fetal Leydig enhancer of the Adrenal 4-binding protein/Steroidogenic factor 1 (Ad4BP/Sf-1, Nr5a1) gene [Shima et al., 2015].

In mice, ALCs emerge approximately 1 week after birth and increase in number until the testis has matured. The major function of ALCs is to supply testosterone and thereby regulate spermatogenesis [Sharpe et al., 1990; Wang et al., 2009]. In addition to the effects on the reproductive organs, testosterone induces secondary male characteristics in many other organs [Macleod et al., 2010]. Considering that in postnatal testes the number of ALCs is substantially higher than that of FLCs, the contribution of FLCs to testosterone production would seem to be insignificant, if present at all. However, because of our limited knowledge regarding the abilities of FLCs, the question remains of whether FLCs play any role in postnatal testes.

The aim of the present study was therefore to determine whether the functions of FLCs in various stages of prenatal and postnatal testes differ by comparing their gene expression profiles.

\section{Materials and Methods}

Preparation of EGFP-Labeled Cells

$m F L E-E G F P$ male mice (previously referred to as $S m A c-1.8$ Ad4BP(LBmut)-EGFP mice) [Shima et al., 2013] were crossed with ICR females (KYUDO). EGFP-positive transgenic testes were harvested at days E14.5 and E18.5 (fetal days) as well as P10, P21, and P56 (postnatal days). Adult Ad4BP-BAC-EGFP mice at 8-10 weeks [Miyabayashi et al., 2015] were used for the preparation of ALCrich cell populations. The EGFP-positive cells were recovered by 2 cycles of fluorescence-activated cell sorting (FACS) (JSAN; Bay Bioscience). Two biologically independent samples $(n=2)$ were prepared for each time point. Total RNA was isolated from the EGFP-positive cells using an RNeasy Micro Kit (Qiagen). Amount and quality of the total RNA were validated using a Bioanalyzer 2100 (Agilent Technologies).

mRNA Sequencing

Poly(A) RNAs were isolated from total RNA samples (50 ng) prepared from the EGFP-positive FLCs at E14.5, E18.5, P10, P21, and P56, and ALCs $(\mathrm{n}=2)$ using a NEBNext Poly $(\mathrm{A})$ mRNA Magnetic Isolation Module (NEB). cDNA libraries for sequencing were constructed using a NEBNext Ultra Directional RNA Library Prep Kit for Illumina (NEB) and NEBNext Multiplex Oligo for Illumina (Index Primer Set 1; NEB). After the quality of the library was validated using the Bioanalyzer 2100, the cDNAs were subjected to sequencing using a HiSeq 1500 (51 bp single-read; Illumina). Sequence data have been deposited in DDBJ/EMBL/GenBank under the accession code DRA004007.

\section{Gene Expression Mapping and Quantification}

Sequence reads were aligned to the reference mouse genome sequence UCSC mm10 (NCBI) using the TopHat program (version 2.0.13) with default parameter settings. Due to the use of transgenic animals ( $m F L E-E G F P$ and Ad4BP-BAC-EGFP mice) for the preparation of FLCs and ALCs, transcripts from the transgene were assumed to hinder the evaluation of the endogenous $A d 4 B P / S f-1$ gene expression. Indeed, we detected transcripts containing the first exon and a part of the second exon of Ad4BP/Sf-1 followed by EGFP in $m F L E-E G F P$ and Ad4BP-BAC-EGFP transgenic mice (online suppl. fig. 1; for all online suppl. material, see www.karger.com/doi/10.1159/000453323). In addition to these transcripts, we unexpectedly found a transcript containing exons 1, 5, 6, and 7 in Ad4BP-BAC-EGFP transgenic mice, and because these transcripts were not detected in the wild type, they were interpreted as being derived from the transgenes. These transcripts were therefore added as reference transcripts to distinguish them from the endogenous transcript. Cufflinks (version 2.2.1) was then used to assemble the transcripts and quantify gene expression (fragments per $\mathrm{kb}$ of transcript per million mapped fragments; FPKM) with default parameter settings.

Detection of Gene Expression Alterations in FLCs and ALCs

Genes whose transcript lengths are $>150$ bp were extracted in order to remove noncoding RNA. Genes with FPKM value differences $>10.0$ between samples (FLCs at E14.5, 18.5, P10, P21, and P56, and ALCs) were extracted. As described by SEQC/MAQC-III Consortium [2014], a pseudo-count of 1 was added to these FPKM values, and then genes with $>2$-fold FPKM differences between the highest and lowest values were further extracted. Using these dif-
54

Sex Dev 2017;11:53-63

DOI: $10.1159 / 000453323$
Miyabayashi/Shima/Inoue/Sato/Baba/

Ohkawa/Suyama/Morohashi 
ferentially expressed genes, cluster analysis was conducted in program R (http://www.R-project.org/) using the function 'hclust' (core distribution), and heatmaps were assembled using the heatmap.2 function of package 'gplots'. The clusters' gene sets were subjected to Gene Ontology (GO) and Kyoto encyclopedia of genes and genomes (KEGG) pathway analyses using the functional annotation tool DAVID (http://david.abcc.ncifcrf.gov/). As described in the following, we detected germ cell contamination in one of the FLC samples at P56. Therefore, the data set from this sample was excluded from the above analyses.

\section{qRT-PCR Analyses}

cDNAs were synthesized from 20 ng of total RNAs ( $n=3$ ) using M-MLV reverse transcriptase (Life Technologies) and random primers. qRT-PCR was performed with a CFX96 real-time PCR system (Bio-Rad) using SYBR Select Master Mix (Applied Biosystems). The expression level off $\beta$-actin (Actb) was used as a control. Primers used for qRT-PCR are listed in online supplementary table 1. Data are presented as means \pm standard deviation (SD).

\section{Results}

\section{Gene Expression of FLCs Altering with Development}

We previously established transgenic mice (FLE-EGFP mice) using the fetal Leydig enhancer (FLE) and the upstream region of the $A d 4 B P / S f-1$ gene [Shima et al., 2012]. These mice provided us with weakly EGFP-labeled cells in addition to strongly EGFP-labeled FLCs [Shima et al., 2013]. Our recent study demonstrated that the former population consists of progenitor cells for FLCs, while the latter consists of differentiated FLCs [Inoue et al., 2016]. In addition to this transgenic mouse line, we established another line in which the LHX9 binding site in the proximal sequence upstream of the $A d 4 B P / S f-1$ gene was mutated (mFLE-EGFP mice) [Shima et al., 2013]. This line was used for FLC preparation in the present study because the mutation resulted in the disappearance of weakly labeled cells. Ad4BP-BAC-EGFP transgenic mice were used in the preparation of ALCs. In this construct, the EGFP expression recapitulates endogenous $A d 4 B P / S f-1$ gene expression because it was prepared using a BAC clone containing the whole $A d 4 B P / S f-1$ gene locus [Miyabayashi et al., 2015].

EGFP-positive FLCs from fetal (E14.5 and E18.5) and postnatal days (P10, P20, and P56) and ALCs were prepared from the transgenic mice described above. Two biologically independent RNA samples at each time point were used for sequencing. An excess of 30 million reads was obtained from every sample, and $93-96 \%$ of reads were mapped to the reference genome (online suppl. table 2). The number of expressed genes was similar between duplicates (online suppl. fig. 2, suppl. table 3).

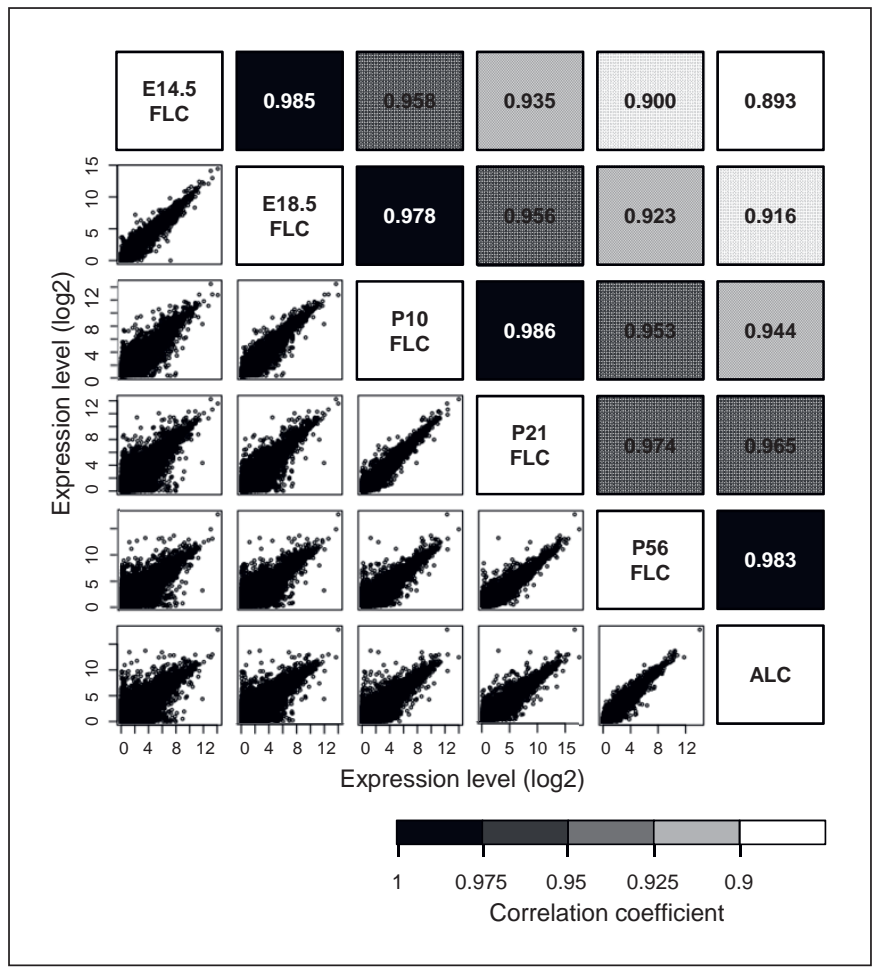

Fig. 1. Comparison of gene expression between various FLCs stages and ALCs. Sequence data sets were obtained from FLCs at E14.5, E18.5, P10, P21, and P56, and from ALCs. Gene expression was compared pairwise between samples. Scatter plots $\left(\log _{2}\right.$ scale) are shown below the diagonal and correlation coefficients above the diagonal, with shading gradients representing coefficient levels. The scale is shown below.

These results indicate a high quality of obtained transcriptomic data sets.

Gene expressions were compared between Leydig cells (fig. 1). The correlation coefficient between neighboring stages was $>0.97$ and decreased with distance between stages. Interestingly, ALCs exhibited the highest correlation coefficient with FLCs at P56, and values decreased gradually with FLCs at earlier stages. These results suggest that FLCs gradually alter their features across developmental stages, until eventually FLCs in adult testis become similar to ALCs.

As described above, the quality of the data sets was sufficient for further analyses. Unfortunately, detailed analyses of the data sets revealed that 1 of the FLC samples at P56 (P56FLC-1) contained a slight germ cell contamination. Although the contamination had low gene expression levels, GO and KEGG pathway analyses resulted in listing germ-related pathways/events. Therefore, P56FLC-1 was excluded from subsequent studies. 


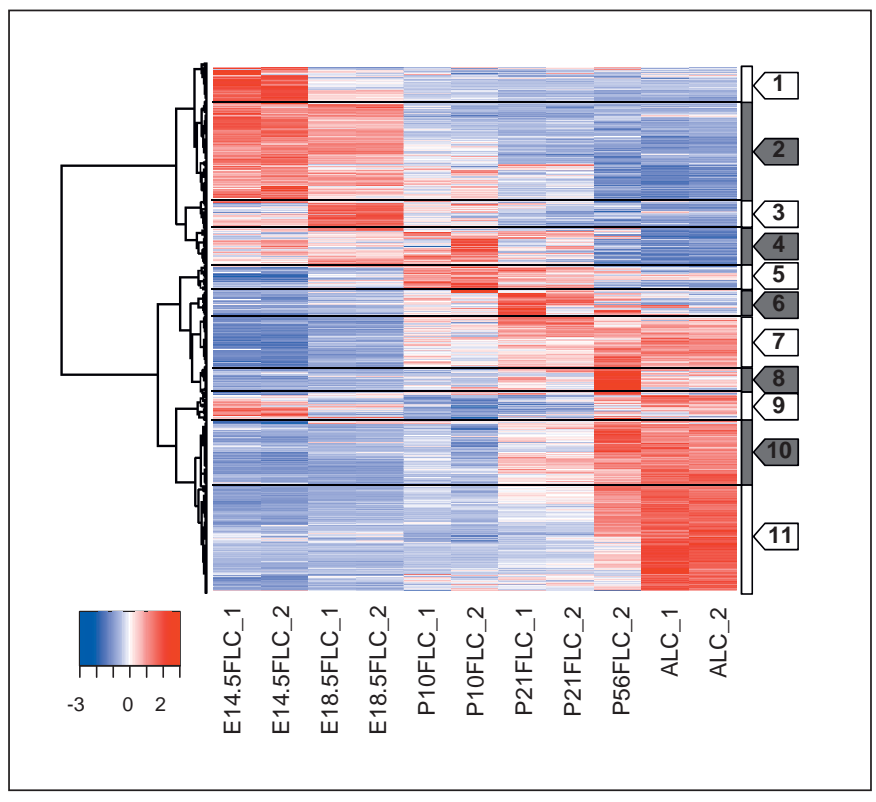

Fig. 2. Heat map of gene expression alterations with development of FLCs. A total of 3,697 genes with maximum expression levels $\geq 2$-fold higher than the minimum level and with FPKM values $\geq 10.0$ were extracted. Transcriptome data sets consisted of 2 RNA samples for FLCs at E14.5, E18.5, P10, P21, and ALCs; a single RNA sample was used for FLCs at P56. Genes were classified into 11 clusters based on expression patterns. Red = Up-regulated; blue $=$ down-regulated. Color shading represents expression change level, and the scale is shown at the lower left.

We attempted to define functional alterations of FLCs across development stages by focusing on the genes with altered expression. A total of 3,697 genes were extracted as described in Materials and Methods. A heat map of these genes also clearly demonstrated that gene expression of FLCs was altering across development stages, and that the expression of FLCs at P56 was most similar to that of ALCs (fig. 2). The genes were classified into 11 clusters (fig. 2), and the gene sets enriched in the clusters (online suppl. tables 4-14) were subjected to GO and KEGG pathway analyses to highlight the biological pathways/events activated in each stage. The classification terms used in the analyses are shown in online supplementary tables 15 (cluster 1 ) to 25 (cluster 11). It is noteworthy that many terms containing 'negative' (such as 'negative regulation of transcription' and 'negative regulation of biosynthetic process') were grouped in clusters 4 and 5 (online suppl. tables 18,19 ). In contrast, many terms containing 'positive' (such as 'positive regulation of transcription' and 'positive regulation of macromolecule biosynthetic process') were grouped in cluster 6 (online suppl. table 20). Many genes classified into clusters 4 and 6 showed the highest expression at P10 and P21, respectively, which strongly suggests that cellular activities of FLCs change substantially during postnatal day 10-21.

\section{Steroidogenesis}

Testosterone production is a fundamental function of the testis in which multiple genes are implicated (fig. 3A). Terms related to steroid metabolism were grouped in cluster 1 (online suppl. table 15) and 9 (online suppl. table 23), indicating that steroidogenic gene expression is more abundant in FLCs at E14.5 and ALCs than in other stages of FLCs. Sequencing and qRT-PCR expression profiles of steroidogenic genes are shown in figure 3B, C. Steroidogenic acute regulatory protein (Star), cholesterol side-chain cleavage P450 (Cyp11a1), 3 $\beta$-hydroxysteroid dehydrogenase 1 (Hsd3b1), and steroid 17a-hydroxylase/ 17,20 lyase P450 (Cyp17a1), all of which are essential for testosterone production, were expressed in FLCs as early as E14.5. As described previously [Shima et al., 2013], Hsd 1763 mediates the final reaction from androstenedione to testosterone and is expressed in Sertoli but not in FLCs in fetal testes. Interestingly, the present study found that $H s d 17 b 3$ was activated in FLCs at P56.

Decreased testosterone synthesis has been established in mice during the early postnatal period [O'Shaughnessy et al., 2002]. During this period, ALCs have not yet matured; hence, this decrease is thought to be due to the decreased testosterone synthetic activity of postnatal FLCs. The decreased expression of Cyp17a1 and Star from the late prenatal to early postnatal period possibly results in the decreased testosterone synthetic activity of the testes. Two paralogues of $H s d 3 b$ genes, $H s d 3 b 1$ and $H s d 3 b 6$, are expressed in mouse gonads. The expression profiles of $H s d 3 b$ s found in the present study strongly suggest that the expression of $H s d 3 b 1$ and $H s d 3 b 6$ is restricted in FLCs and ALCs, respectively. This preferential expression of the $2 \mathrm{Hsd} 3 \mathrm{bs}$ is consistent with published observations [Baker et al., 1999; O’Shaughnessy et al., 2002].

Steroid 21-hydroxylase P450 (Cyp21a1) and steroid $11 \beta$-hydroxylase P450 (Cyp11b1), established as markers for the adrenal cortex, are essential for adrenocorticoid production but not for sex steroids. We unexpectedly detected expressions of these genes in FLCs at the fetal ages, although the FPKM values were somewhat lower than those of Cyp11a1 and Cyp17a1. The adrenocortical marker gene expression in FLCs, even at lower levels, may reflect the fact that the gonads and adrenal cortex originate from a single cellular population, the adreno-gonadal primordium [Hatano et al., 1996]. $\begin{array}{ll}56 & \text { Sex Dev } 2017 ; 11: 53-63 \\ & \text { DOI: } 10.1159 / 000453323\end{array}$
Miyabayashi/Shima/Inoue/Sato/Baba/ Ohkawa/Suyama/Morohashi 
Fig. 3. Steroidogenic gene expression in FLCs and ALCs. A Steroid synthetic pathways in the gonad and adrenal cortex with genes and intermediates/final products. B mRNA sequencing-derived expression profiles of the steroidogenic genes Star, Cyp11a1, Hsd3b1, Cyp17a1, Hsd17b3, Hsd3b6, Cyp21a1, and Cyp11b1. Two FLC samples from E14.5, E18.5, P10, and P21, 2 ALC sample from P56, and a single FLC sample from P56 were used. Genes classified into cluster 9 are enclosed by a dashed line. C The expressions of Star, Cyp11a1, $H s d 3 b 1, C y p 17 a 1, H s d 17 b 3$, and Hsd3b6 were examined by qRT-PCR with 3 biologically independent samples $(\mathrm{n}=3)$. Actb was used as the qRT-PCR standard.

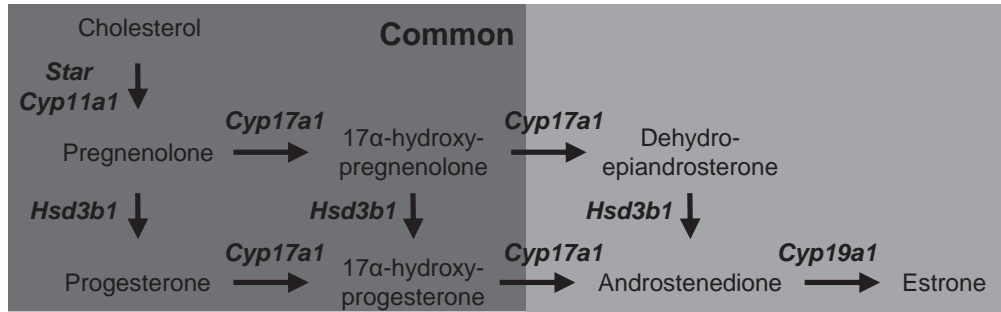

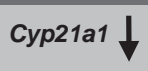$$
\text { (21a1 }
$$

Hsd17b3 \

11-Deoxy- Cyp17a1 1 -Deoxycortisol Testosterone 17ß-Estradiol

Hsd17b3 \ icosterone

Cyp11b1

Cyp11b1

Corticosterone

Cortisol

Cyp11b2 \

A

Aldosterone

Adrenal

Gonad

\section{mRNA-seq}
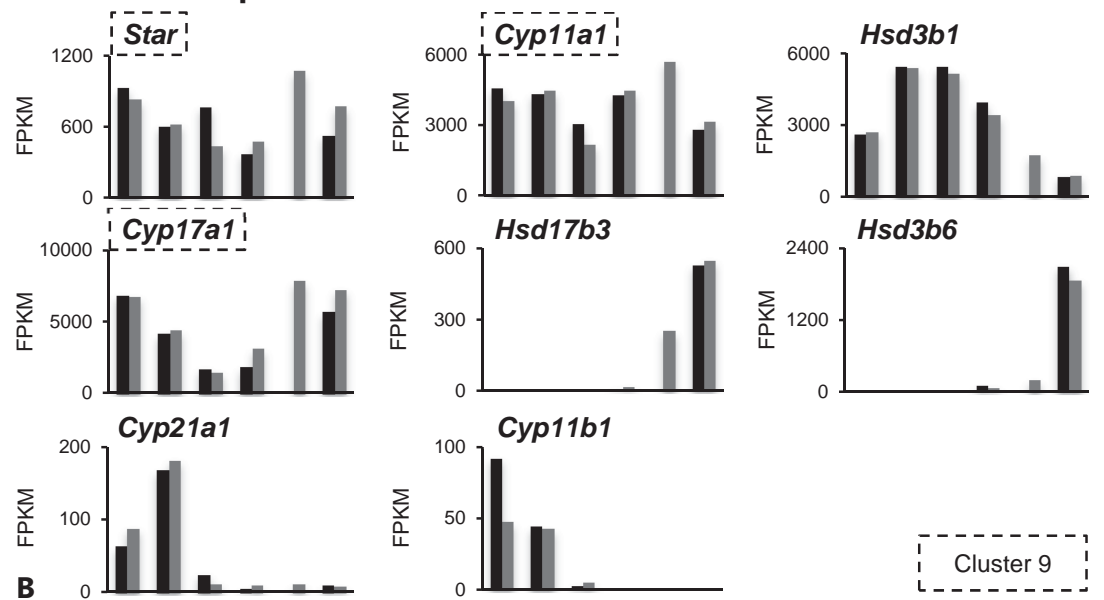

qRT-PCR
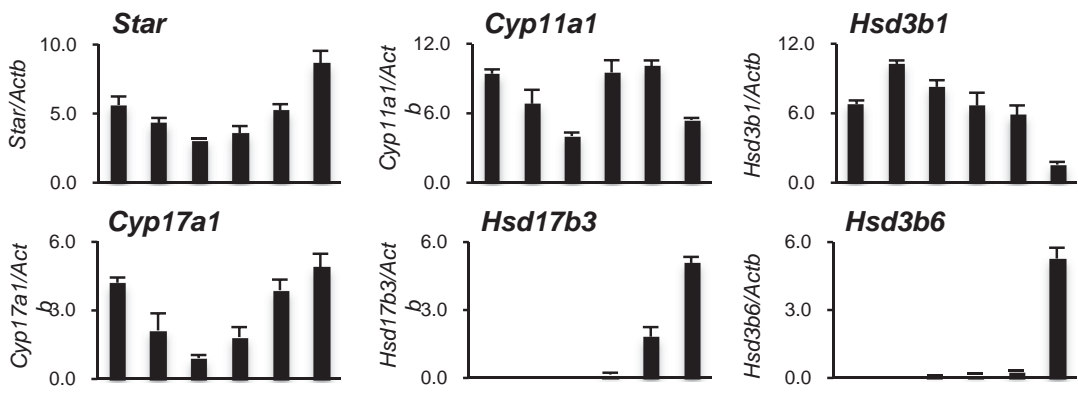

Hsd17b3

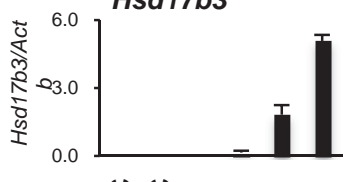

00

藏 0 ○ 0

เ

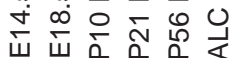

1 000

เ

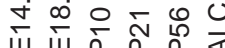

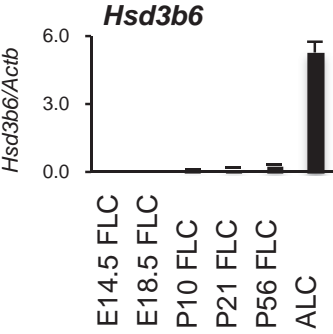




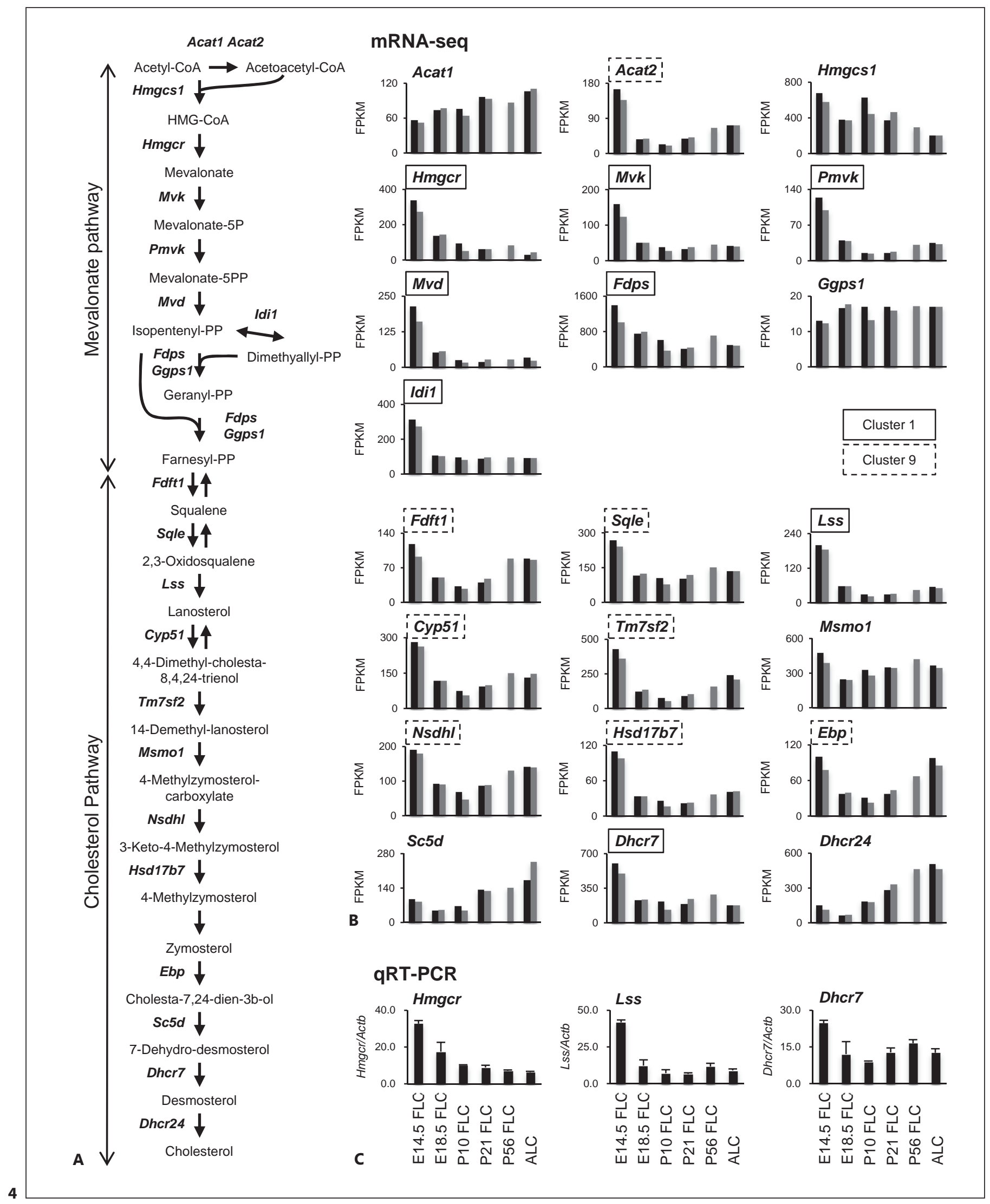

(For legend see next page.) 


\section{Cholesterogenesis}

Similar to steroid metabolism, terms related to cholesterol metabolism were grouped in clusters 1 (online suppl. table 15) and 9 (online suppl. table 23). It is reasonable that both steroidogenesis and cholesterogenesis were grouped into the same clusters since steroid hormones are synthesized from cholesterol. The expressions of all cholesterogenic genes revealed by sequencing and qRT-PCR are shown in figure 4 . The cholesterol synthetic pathway can be divided into the mevalonate pathway and the cholesterol pathway. The mevalonate pathway results in the production of farnesyl diphosphate, which is utilized for the synthesis of isoprenoid as well as cholesterol (cholesterol pathway) [Santos and Lehmann, 2004; Tricarico et al., 2015]. Notably, many genes in the mevalonate pathway were grouped in cluster 1, with the highest expression at E14.5 (solid boxes in fig. 4B). In contrast, the genes in the cholesterol pathway were grouped in cluster 9 , with the highest expression at E14.5, while the expressions in FLC at P56 and ALCs were higher than at other FLCs stages (dashed boxes in fig. 4B). Expression profiles obtained by sequencing were validated by qRT-PCR for 3-hydroxy3-methylglutaryl-CoA reductase $(\mathrm{Hmgcr})$, lanosterol synthase (Lss), and 7-dehydrocholesterol reductase (Dhor7) (fig. 4C).

\section{Mitochondrial Biogenesis and Functions}

Several terms related to mitochondrial structures and functions were grouped in clusters 7 (online suppl. table 21), 8 (online suppl. table 22), 10 (online suppl. table 24), and 11 (online suppl. table 25), suggesting that mitochondrial biogenesis and functions become active postnatally. For mitochondrial functions, terms related to lipid metabolism and oxidative phosphorylation are included. As shown in figure 5A, many genes necessary for the import of fatty acids into mitochondria and mitochondrial $\beta$-oxidation are implicated in lipid metabolism.

Fig. 4. Cholesterogenic gene expression in developing FLCs and ALCs. A Cholesterogenic pathway with genes and intermediate products, divided into the mevalonate pathway and the cholesterol pathway. B Sequencing-derived gene expression profiles. Genes involved in the mevalonate pathways are shown in the upper part, genes involved in the cholesterol pathway are shown in the lower part. Genes classified into clusters 1 and 9 are enclosed by solid and dashed lines, respectively. C qRT-PCR-derived expressions of $H m g c r, L s s$, and Dhcr7 (n = 3). Actb was used as the qRT-PCR standard.

Fetal Leydig Cell Gene Expression during Development
Many of these gene expressions were higher in postnatal than prenatal FLCs, and expressions in ALCs were mostly higher than in FLCs (fig. 5B).

Oxidative phosphorylation (OXPHOS) mediated by protein complexes I-V, which ultimately generates ATP, is another fundamental function of the mitochondrion (fig. 6A). A large number of genes are implicated in the formation of these complexes. Expression profiles of a part of OXPHOS genes are shown in figure 6B. All these gene expressions were increased in FLCs from fetal to adult stages. qRT-PCR-derived expression profiles were similar to those obtained by sequencing. Many of these OXPHOS genes were highly expressed in ALCs (fig. 6C).

Taking into account elevated oxygen tension after birth [Heymann and Rudolph, 1972], the expression of Hifl $\alpha$ was assumed to decrease at that point. As expected, expression during the postnatal period was lower than that during the prenatal period (fig. 6D). Peroxisome proliferator-activated receptor- $\gamma$ coactivator 1 (PGC-1) family members regulate mitochondrial biogenesis [Schönenberger and Kovacs, 2015]. Unexpectedly, the expression levels of $P g c-1 \alpha, P g c-1 \beta$, and the genes structurally related to $P g c-1$ were quite low and did not show any alteration.

\section{Discussion}

Mammals develop 2 types of Leydig cells, FLCs and ALCs. In contrast, there has been no report indicating the presence of cells corresponding to FLCs in non-mammalian taxa [Griswold and Behringer, 2009; Teerds and Huhtaniemi, 2015]. The physiological function of FLCs has therefore generally been discussed in relation to the reproductive system present in mammals. The prominent feature of mammalian reproduction is that the embryo undergoes its whole developmental process in utero, and thus male fetuses are forced to be masculinized in an abundantly estrogenic milieu. Accordingly, it has been proposed that the appearance of FLCs guarantees the development of male features even in the estrogenic milieu by activating robust androgen production [Huhtaniemi and Pelliniemi, 1992]. Given that the major role of FLCs is androgen production during the fetal period, it could be assumed that they would disappear soon after birth. A recent study, however, clearly demonstrated the persistent presence of FLCs in adult testis [Shima et al., 2015]. To characterize the functions of prenatal to postnatal FLCs, we examined the gene expression of FLCs at multiple stages.

Sex Dev 2017;11:53-63

DOI: $10.1159 / 000453323$ 


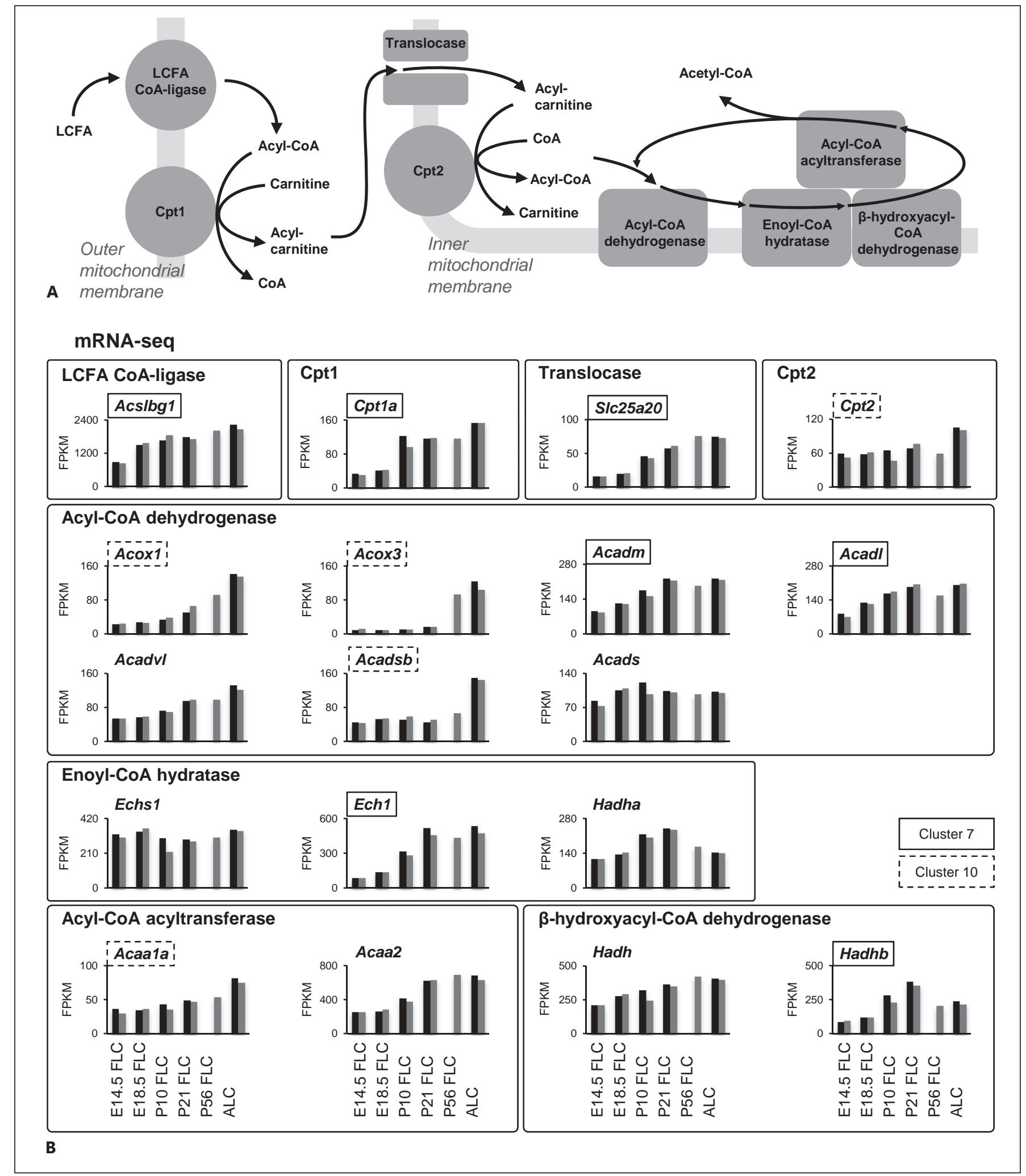

Fig. 5. Expression of genes implicated in lipid metabolism. A Lipid transport from cytosol into mitochondria and mitochondrial $\beta$-oxidation. LCFA = Long-chain fatty acid; $\mathrm{Cpt}=$ carnitine palmitoyl transferase. $\mathbf{B}$ Sequencing-derived gene expression profiles. Genes classified into clusters 7 and 10 are enclosed by solid and dashed lines, respectively. 
Fig. 6. Expression of genes implicated in OXPHOS. A Schematic representation of OXPHOS complexes (complexes I-V) localized in the mitochondrial inner membrane. B Sequencing-derived expression profiles of OXPHOS genes. Genes classified into cluster 11 are enclosed by a solid line. C qRT-PCR-derived expressions of $N d u f a 6$ and Ndufs8 in complex I, Sdhb in complex II, Uqcr10 and Uqcrc1 in complex III, Cox6c and Cox8a in complex IV, and Atp5e and Atp5l in complex V $(\mathrm{n}=3)$. Actb was used as the qRT-PCR standard. D Sequencing-derived expression of Hifla.
A

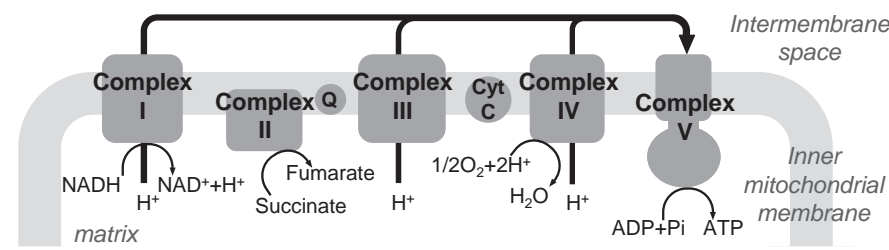

mRNA-seq

Cluster 11

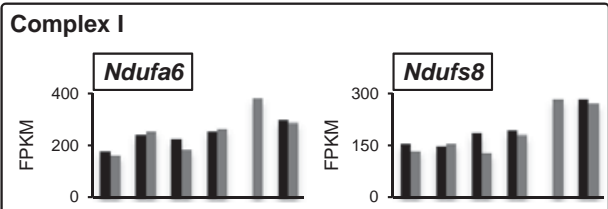

Complex II
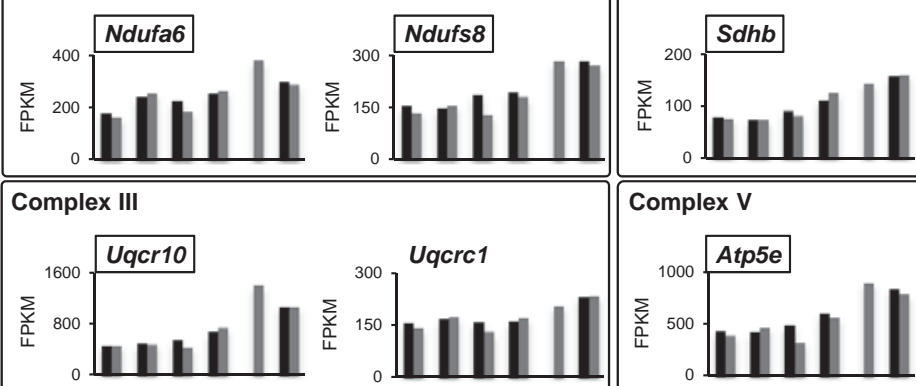

Complex V

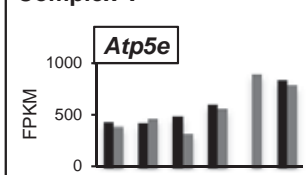

Complex IV
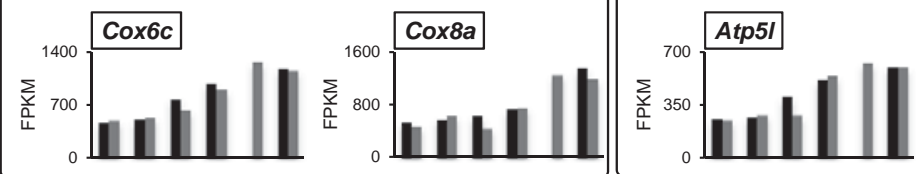

qRT-PCR

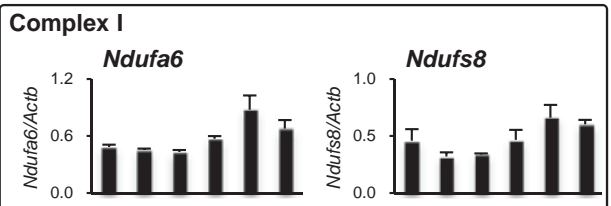

Complex II

Sdhb

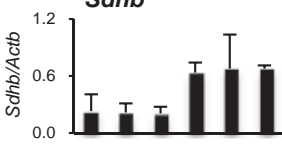

Complex III

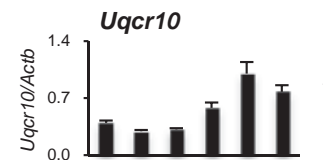

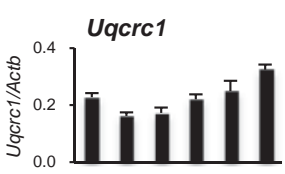

Complex V

Atp5e

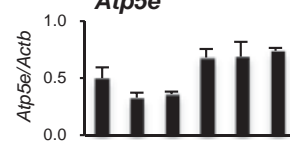
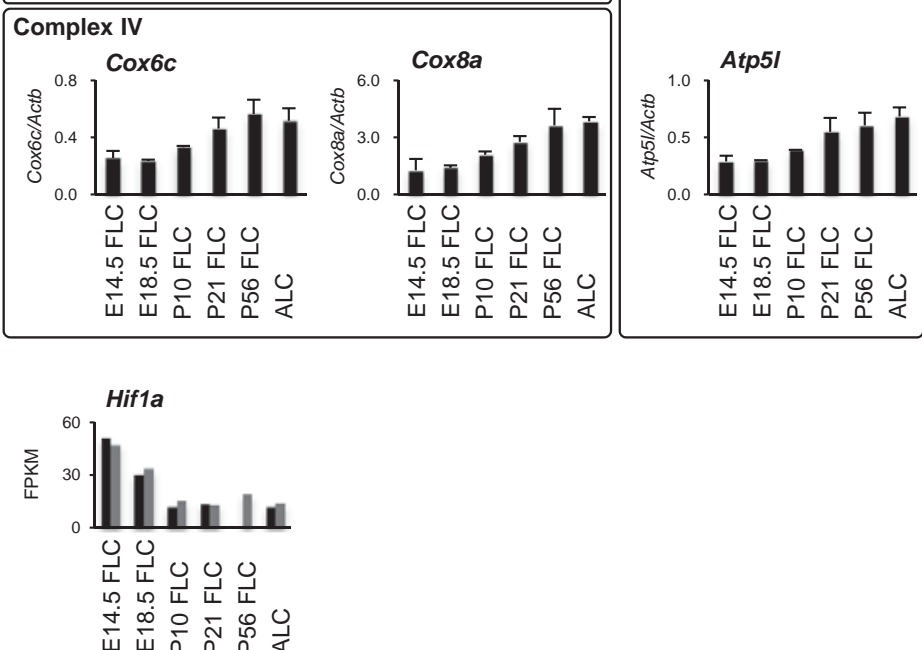

Fetal Leydig Cell Gene Expression during Development 
Altered Gene Expression in FLCs Potentially due to Environmental Changes

The present study demonstrates that the gene expression of FLCs gradually alters across developmental stages. FLCs in the adult testes were shown to mostly resemble ALCs, while FLCs at E14.5 mostly differ from ALCs. GO and KEGG pathway analyses revealed that genes with abundant expression in FLCs at P10 are related to the suppression of various cellular activities, whereas those abundant in FLCs at P21 are related to cellular activation. These contrasts strongly suggest that the biological activities of FLCs alter substantially during the postnatal period.

While oxygen is supplied to the fetus by the mother via umbilical cord blood, postnatally it is taken up by the neonates' respiratory system. This change results in an increase in oxygen tension in the neonate [Heymann and Rudolph, 1972]. Likewise, nutrients are supplied by the mother through the cord blood during the fetal period and through milk during the neonatal period. Young mice start to ingest solid food approximately 2 weeks after birth. This has led to the assumption that extensive changes in oxygen and nutrient status occur during the early postnatal period. The alteration of gene expression observed in postnatal FLCs may be partially or entirely related to these physiological changes.

In this regard, it should be noted that hypoxia inducible factor-1a $(\mathrm{Hif} 1 \alpha)$, which is activated under hypoxia conditions, suppresses OXPHOS gene expression. Hence, postnatal increase of oxygen tension promotes mitochondrial biogenesis through decreased Hifl $\alpha$ expression [Schönenberger and Kovacs, 2015]. The present study found that the expression of Hif $1 \alpha$ is decreased from fetal to adult stages, showing an inverse correlation with mitochondrial functional genes, OXPHOS, and fatty acid $\beta$-oxidation. The alteration of Hifl $\alpha$ gene expression from prenatal to postnatal FLCs may induce these mitochondrial genes in response to the increased oxygen tension.

\section{Comparison of Findings with Previously Published}

Data

Several studies using microarray or deep-sequencing to investigate gene expression of cells in fetal testes have been published [Jameson et al., 2012; McDowell et al., 2012; McClelland et al., 2015]. In these studies, Sertoli cells, germ cells, FLCs, and interstitial cells from transgenic mice carrying Sox9-EGFP, Mafb-EGFP, Oct4$m$ Cherry, or SF1-BAC-EGFP as the transgenes were fractionated by FACS. However, we suggest that it would be difficult to obtain pure fractions of FLCs unless our transgenic mouse line (FLE-EGFP) is used. The purity of the cell population may be critical for large scale analyses by microarray and next-generation sequencing. In the present study, one of the FLC samples at P56 was found to contain a germ cell contamination even with careful preparation. Although the contamination was likely to be very low, comparison of the gene expression with other stages of FLCs resulted in the highlighting of genes expressed in the contaminated germ cells. As a consequence, GO and KEGG pathway analyses readily resulted in erroneous findings when listing biological events/pathways specific for germ cells. These terms disappeared when the compromised data set was excluded from the analyses, highlighting that the purity of the sample is critical for this type of study.

While many studies have so far concluded that FLCs and ALCs are different in terms of gene expression, these studies were based on comparisons between FLCs in fetal testes and ALCs in adult testes. Similar to these studies, the present study found many genes with substantial differences in expression between fetal FLCs and adult ALCs. In addition, our study investigated various stages of FLCs to reveal for the first time that FLCs alter the expression of metabolic genes from prenatal to postnatal periods and become similar to ALCs in their gene expression.

\section{Acknowledgments}

This work was supported by the Research Support Center, Graduate School of Medical Sciences, Kyushu University. This work was also supported by the Japan Society for the Promotion of Science (JSPS) KAKENHI Grant Numbers 21249018, $16 \mathrm{H} 05142$ (K. Morohashi), and 25116010 (Y.O.), and the Ministry of Education, Culture, Sports, Science, and Technology, Japan (MEXT) KAKENHI Grant Number 22132002 (K. Morohashi), AMEDCREST, JST (Y.O., M.S.), The Uehara Memorial Foundation, and Takeda Science Foundation.

\section{Statement of Ethics}

All animal experimental protocols were approved by the Animal Care and Use Committee of Kyushu University.

\section{Disclosure Statement}

The authors have no conflicts of interest to declare.
62

Sex Dev 2017;11:53-63

DOI: $10.1159 / 000453323$
Miyabayashi/Shima/Inoue/Sato/Baba/ Ohkawa/Suyama/Morohashi 


\section{References}

Baker PJ, Sha JA, McBride MW, Peng L, Payne AH, O'Shaughnessy PJ: Expression of $3 \beta$-hydroxysteroid dehydrogenase type I and type VI isoforms in the mouse testis during development. Eur J Biochem 260:911-917 (1999).

Brennan J, Capel B: One tissue, two fates: molecular genetic events that underlie testis versus ovary development. Nat Rev Genet 5:509-521 (2004).

Brennan J, Tilmann C, Capel B: $P d g f r-\alpha$ mediates testis cord organization and fetal Leydig cell development in the XY gonad. Genes Dev 17: 800-810 (2003).

Buaas FW, Gardiner JR, Clayton S, Val P, Swain A: In vivo evidence for the crucial role of SF1 in steroid-producing cells of the testis, ovary and adrenal gland. Development 139:45614570 (2012).

Cui S, Ross A, Stallings N, Parker KL, Capel B, Quaggin SE: Disrupted gonadogenesis and male-to-female sex reversal in Pod1 knockout mice. Development 131:4095-4105 (2004).

Griswold SL, Behringer RR: Fetal Leydig cell origin and development. Sex Dev 3:1-15 (2009).

Hatano O, Takakusu A, Nomura M, Morohashi $\mathrm{K}$ : Identical origin of adrenal cortex and gonad revealed by expression profiles of Ad4BP/ SF-1. Genes Cells 1:663-671 (1996).

Heymann MA, Rudolph AM: Effects of congenital heart disease on fetal and neonatal circulations. Prog Cardiovasc Dis 15:115-143 (1972).

Huhtaniemi I, Pelliniemi LJ: Fetal Leydig cells: cellular origin, morphology, life span, and special functional features. Proc Soc Exp Biol Med 201:125-140 (1992).

Inoue M, Shima Y, Miyabayashi K, Tokunaga K, Sato T, Baba T, et al: Isolation and characterization of fetal Leydig progenitor cells of male mice. Endocrinology 157:1222-1233 (2016).

Jameson SA, Natarajan A, Cool J, DeFalco T, Maatouk DM, et al: Temporal transcriptional profiling of somatic and germ cells reveals biased lineage priming of sexual fate in the fetal mouse gonad. PLoS Genet 8:e1002575 (2012).

Kerr JB, Knell CM: The fate of fetal Leydig cells during the development of the fetal and postnatal rat testis. Development 103:535-544 (1988).

Kuopio T, Tapanainen J, Pelliniemi LJ, Huhtaniemi I: Developmental stages of fetal-type Leydig cells in prepubertal rats. Development 107:213-220 (1989).
Macleod DJ, Sharpe RM, Welsh M, Fisken M, Scott HM, et al: Androgen action in the masculinization programming window and development of male reproductive organs. Int J Androl 33:279-287 (2010).

McClelland KS, Bell K, Larney C, Harley VR, Sinclair AH, et al: Purification and transcriptomic analysis of mouse fetal Leydig cells reveals candidate genes for specification of gonadal steroidogenic cells. Biol Reprod 92:145 (2015).

McDowell EN, Kisielewski AE, Pike JW, Franco $\mathrm{HL}$, Yao $\mathrm{HH}$, Johnson KJ: A transcriptomewide screen for mRNAs enriched in fetal Leydig cells: CRHR1 agonism stimulates rat and mouse fetal testis steroidogenesis. PLoS One 7:e47359 (2012)

Meeks JJ, Crawford SE, Russell TA, Morohashi K, Weiss J, Jameson JL: Dax1 regulates testis cord organization during gonadal differentiation. Development 130:1029-1036 (2003).

Miyabayashi K, Katoh-Fukui Y, Ogawa H, Baba $\mathrm{T}$, Shima Y, et al: Aristaless related homeobox gene, Arx, is implicated in mouse fetal Leydig cell differentiation possibly through expressing in the progenitor cells. PLoS One 8:e68050 (2013).

Miyabayashi K, Tokunaga K, Otake H, Baba T, Shima Y, Morohashi K: Heterogeneity of ovarian theca and interstitial gland cells in mice. PLoS One 10:e128352 (2015).

O’Shaughnessy PJ, Baker PJ, Heikkilä M, Vainio S, McMahon AP: Localization of $17 \beta$ hydroxysteroid dehydrogenase/17-ketosteroid reductase isoform expression in the developing mouse testis - androstenedione is the major androgen secreted by fetal/neonatal Leydig cells. Endocrinology 141:2631-2637 (2000).

O’Shaughnessy PJ, Willerton L, Baker PJ: Changes in Leydig cell gene expression during development in the mouse. Biol Reprod 66:966975 (2002)

Santos AC, Lehmann R: Isoprenoids control germ cell migration downstream of HMGCoA reductase. Dev Cell 6:283-293 (2004).

Schönenberger MJ, Kovacs WJ: Hypoxia signaling pathways: modulators of oxygen-related organelles. Front Cell Dev Biol 3:42 (2015).
SEQC/MAQC-III Consortium: A comprehensive assessment of RNA-seq accuracy, reproducibility and information content by the Sequencing Quality Control Consortium. Nat Biotechnol 32:903-914 (2014).

Sharpe RM, Maddocks S, Kerr JB: Cell-cell interactions in the control of spermatogenesis as studied using Leydig cell destruction and testosterone replacement. Am J Anat 188:3-20 (1990).

Shima Y, Miyabayashi K, Baba T, Otake H, Katsura $\mathrm{Y}$, et al: Identification of an enhancer in the $A d 4 B P / S F-1$ gene specific for fetal Leydig cells. Endocrinology 153:417-425 (2012).

Shima Y, Miyabayashi K, Haraguchi S, Arakawa $\mathrm{T}$, Otake H, et al: Contribution of Leydig and Sertoli cells to testosterone production in mouse fetal testes. Mol Endocrinol 27:63-73 (2013).

Shima Y, Matsuzaki S, Miyabayashi K, Otake H, Baba T, et al: Fetal Leydig cells persist as an androgen-independent subpopulation in the postnatal testis. Mol Endocrinol 29:15811593 (2015).

Svingen T, Koopman P: Building the mammalian testis: origins, differentiation, and assembly of the component cell populations. Genes Dev 27:2409-2426 (2013)

Tang H, Brennan J, Karl J, Hamada Y, Raetzman L, Capel B: Notch signaling maintains Leydig progenitor cells in the mouse testis. Development 135:3745-3753 (2008).

Teerds KJ, Huhtaniemi IT: Morphological and functional maturation of Leydig cells: from rodent models to primates. Hum Reprod Update 21:310-328 (2015).

Tricarico PM, Crovella S, Celsi F: Mevalonate pathway blockade, mitochondrial dysfunction and autophagy: a possible link. Int J Mol Sci 16:16067-16084 (2015).

van den Driesche S, Walker M, McKinnell C, Scott HM, Eddie SL, et al: Proposed role for COUP-TFII in regulating fetal Leydig cell steroidogenesis, perturbation of which leads to masculinization disorders in rodents. PLoS One 7:e37064 (2012).

Wang RS, Yeh S, Tzeng CR, Chang C: Androgen receptor roles in spermatogenesis and fertility: lessons from testicular cell-specific androgen receptor knockout mice. Endocr Rev 30: 119-132 (2009).

Yao HH, Whoriskey W, Capel B: Desert Hedgehog/Patched 1 signaling specifies fetal Leydig cell fate in testis organogenesis. Genes Dev 16 : 1433-1440 (2002). 